\title{
Bias in Geographic Information Systems: The Case of Google Maps
}

\author{
Ben Wagner \\ Faculty of Technology \\ Policy and Management \\ TU Delft \\ ben@benwagner.org
}

\author{
Till Winkler \\ Institute for Information \\ Systems and Society, \\ Vienna University of \\ Economics and Business, Austria \\ till.winkler@wu.ac.at
}

\author{
Soheil Human \\ Institute for Information Systems \\ and New Media, \\ Vienna University of \\ Economics and Business, Austria \\ soheil.human@wu.ac.at
}

\begin{abstract}
Users' perception of geographic space depends heavily on geographic information systems (GIS). GIS are the most common way for users to estimate travel time, provide routing information and recommend appropriate forms of transportation. This article analyses how predictions made by Google Maps, one of the most popular GIS, influence users' perceptions and travel choices. To analyze this influence, a pre-study in a classroom setting $(n=36)$ as well as an online survey $(n=521)$ were conducted. We study users intuitive perception of travel time, before using the Google Maps Mobile App as a 'treatment' to see how it influences their perceptions of travel time and choice of transportation type. We then contrast this original Google Maps treatment to a mock-up 'warning label version' of Google which informs users about biases in Google Maps and an 'unbiased version' of Google Maps based on ground truth data. Our analysis suggests that Google Maps systematically underestimates necessary car driving time, which has an impact on users' choice of transportation.
\end{abstract}

\section{Introduction}

The perception of geographic reality depends heavily on the tools, such as paper maps or geographic information systems (GIS) used to perceive it $[1,2$, $3,4]$. Developing a map involves countless subjective decisions [5] and provides a window into the world-view of its creator $[1,6,7]$. Especially when a system is implemented on a large scale [8], subjective decisions are typically invisible to users and can result in systematic biases [9]. In other words, a perfect map does not exist [5]; rather, a map or navigation system can be more or less appropriate for certain tasks or contexts. Navigation systems can influence how users perceive environmental distance [10] and influence spatial behavior [11]. This means that the way a GIS-such as Google Maps which has about $68 \%$ market share-presents information influences which form of transportation users consider to be the best option. Importantly even without any tools human beings are not able to perfectly conceptualize geographic space accurately, and there are numerous well documented distortions in how human beings perceive spatial relationship [12] and distance [10]. This is why tools such as GIS are so important, as they are tailored to improve users' ability to make accurate predictions about their surroundings (i.e. to empower users $[13,14])$. When interpreting their surroundings, users rely on their individual cognitive models of geographic space $[15,3]$ as well as of the information system they are using to support this mapping [16]. However as there is no such thing as a perfect map [5], in some use cases the influences on users behavior may not necessarily be as the creator intended. At the same time it should be acknowledged that users themselves are not the best at making estimates and frequently misinterpret how long a trip actually took them $[17,18,19,20]$. We conceptualize this difference between a 'perfect map' based on ground truth, which refers to field exploration in cartography, and the predictions made by Google Maps as system bias [9]. Furthermore, we define user bias as the difference between ground truth and predictions made by users on how long a route will take. In doing so a clear differentiate between system bias and user bias is possible. Ground truth data was collected by the researchers by traveling on the actual routes and measuring door to door transportation time for the routes presented to users in a mockup setting. The presented 
case study captures both biases in Vienna as a particular urban setting.

The case study analyses the ways in which users perceive the recommendations they receive from Google Maps in Vienna and how these influence their spatial perception. Furthermore, we study to what extent users trust the recommendations they receive from Google Maps and how this influences their chosen mode of transportation. This will be contrasted with users intuitive perception of travel time and choice of transportation used. In doing so we attempt to understand the following research questions:

RQ 1: Are there any algorithmic bias in Google Map when used in Vienna? To answer this research question, we analysed how information is presented to users in the Android version of the Google Maps mobile app [21]. Our analysis suggests that in central Vienna, Google Maps systematically presents driving trip time differently than public transport trip time, by including some elements such as the process of walking to public transport or walking from public transport to the final destination that are not included in driving trip time. We believe that in an urban setting where parking is rarely available exactly at the destination, this will lead to Google Maps underestimating the total driving trip time. Based on this analysis and the assumption that users will be influenced by shorter predicted driving times, we suspect that as a result users will be more likely to select driving as their preferred option.

RQ 2: How strong is the effect of Google Maps on how users perceive travel time in Vienna? Based on the previous analysis, we conducted a pre-study with students $(n=36)$ and an online survey $(n=521)$. In the online survey, we tested three potential travel routes in Vienna. We compared the intuitive answers of users for best type of transportation with answers given after using an interactive mock-up of the original Android version of Google Maps.

RQ 3: How strong is the effect of Google Maps on users' choice of best type of transportation in Vienna? Analysis, pre-study and the online survey all suggest that Google Maps has an effect on estimated travel time of users. Specifically after using Google Maps, users of Google Maps tend to be more likely to underestimate car driving travel time. In order to assess the strength of the effect we tested two accountability mechanisms [22, 23, 24], warning label version and unbiased version, to see whether users when informed about bias in Google Maps or when presented with more accurate data would make different travel decisions.

It is important to acknowledge that biases can also exist in other similar geographic information systems. We focus on Google Maps here as it is one of the largest mapping tools of this kind, however other tools also exhibit similar biases and limitations in specific contexts. The next chapter introduces the utilized research methodology and an explanation is given regarding how biases are operationalized. Next, the results of the analysis, pre-study and online survey are presented and discussed. Finally, the conclusion addresses the limitations of this paper as well as pathways for future research.

It has been proposed that information systems should be human-centric [25] and pluralist [26, 27], i.e to continuously consider their users' diverse abilities, limits, needs [28] and values [26]. It is, however, imperative that systems be accountable, at a minimum, to their users. As a result of automation bias [29, 30], users are overly reliant on automated systems, making the accountability of these systems particularly important. Therefore, we have additionally measured and analyzed the extent to which users trust Google Maps, whether this trust is warranted and how the two proposed accountability mechanisms impact users' trust in the system $[29,30]$.

By answering these research questions, this paper makes a contribution to the discussion on the effects of bias in technical systems on the end users of those systems. Furthermore, it provides insights into the biases of Google Maps and shows how these influence the perceptions and decisions of users. While our results are limited to Vienna, which has a well-developed public transport system, the methodological approach can serve as a foundation for future work into the effects of biases on users and allows the testing of other potential accountability mechanisms. Furthermore, this paper shows a pathway to design effective accountability mechanisms. As our results reveal that the bias in Google Maps potentially nudges users towards choosing automobiles, this paper could additionally advance the discussion on the environmental impact of navigation systems.

\section{Methodology}

To answer our three research questions, we used a three-step approach: analysis, pre-study and online survey. In this chapter, these three steps are explained in detail.

As a first step, we conducted an analysis of the Google Maps Android App, based on systematic testing of the software in Vienna and a comparison to other GIS applications. This enabled us to develop an understand on which aspects the routing algorithm considers and what aspects it ignores. This also allowed us to answer the first question (RQ 1), providing a clear overview of 
the bias in Google Maps and serving as the basis for subsequent steps.

As a second step, we conducted a small pre-study involving students $(n=36)$ participating in a masters level course on user-centric design on 8 June 2018. This allowed us to begin to answer the second question (RQ2) and understand the potential effects of biases $(\Delta$ System) on perceived travel time $(\Delta$ User $)$ as well as users transportation choices. Another aim was to understand on what basis students make their intuitive estimations of travel time and transportation choices. The results of the pre-study discussion were recorded using the survey tool Mentimeter.

In a third step, we developed an online survey ( $n=521)$ utilizing image maps as clickable mockups to answer the third research question (RQ3). We recruited participants through an email list that includes all students of the university who have not chosen to unsubscribe. Participants were randomly assigned to one of three mockup conditions and presented with three routes each. This routes were chosen based on our previous analysis, for which a strong bias was likely to be present. All routes started at the university and went to well-known locations within the city of Vienna: a bar along the Danube canal (route 1), a large flak tower in the middle of a public park (route 2) and a famous architectural site (route 3 ). The distance to all of these locations is relatively short $(2-4 \mathrm{~km})$, which make other types of transportation, for instance, walking or biking, a feasible alternative. Additionally, Vienna has a well-developed and inexpensive public transport system, which makes public transport a reasonable option on all of these routes.

For each route, participants where first asked to give their intuitive travel time estimation and choice of transportation type $(\alpha)$. Afterwards they were presented with one of three mock-up conditions and asked to choose the mode of transportation they considered to be the best. A comparison of the answers before and after seeing the mock-up for each route allows us to analyze how strong the effect of Google Maps is on users' choice of best type of transportation (RQ3). The online survey follows a between-subjects design, according to which each participant is randomly assigned to one of three mock-up conditions. Comparing the results between the three conditions allows us to to determine the effectiveness of the two proposed accountability mechanisms and what effect they have on the chosen mode of transportation and users' trust in Google Maps.

\section{Treatment Design}

Additionally, at the beginning and end of the survey the users were asked how much experience they had using Google Maps and whether they believe it has accurate mapping data, suggests good routes and can be trusted for navigation. Moreover, for each transportation mode decision, the users had to justify their choice. This information provided by users can give us insights on whether users know about biases in Google Maps $(\gamma)$. The different possible decisions are:

\subsection{Original Version}

Participants in the first condition saw an original version of Google Maps as can be seen in figure 1. This was presented as a clickable mockup based on screenshots from the Android App, taken between $6-8 \mathrm{pm}$ for each route. This condition helps to answer RQ3 and acts as a control for the two proposed accountability mechanism. Figure 1 shows the used mockup screenshots.

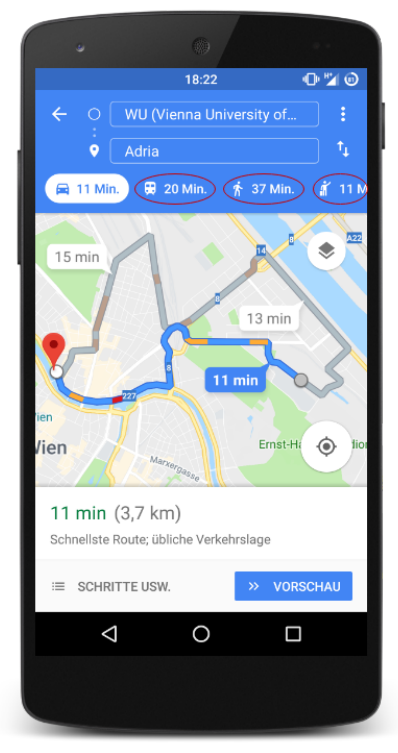

Figure 1. Original version of Google Maps for driving by car

\subsection{Unbiased Version}

In the second condition, participants saw an unbiased version of Google Maps. This possible accountability 
mechanism rectifies all incorrect assumptions from the Google Maps estimates for all routes. To correct for existing system biases and be able to create an unbiased version of Google Maps, "ground truth" data were collected. We achieved this by collecting Google Maps recommendations $(\beta)$ for all routes and drove them on the evening of 2 August 2018 in Vienna. This was done between 6-8pm to avoid any significant divergence based on traffic or similar issues. Additionally, we corrected for errors in public transport time estimates and taxi service arrival predictions. We did not correct walking distance data or cycling data, as these were accurate in these cases according to our analysis. This "ground truth" data allowed us to develop an estimate for the system biases in Google Maps for these routes. We compared these "ground truth" data, which represent real world travel time, to the predictions made by Google Maps for the same route ( $\Delta$ System). On a practical level, the original Google Maps screenshots were edited using Gimp to present the participants with a corrected clickable mockup.

\subsection{Warning Label Version}

For the third condition, participants saw the original version of Google Maps, but with warning labels. The first screenshot the participants saw introduced the warning signs, which they had to acknowledge. Afterwards, for each route there was a clickable warning sign that informed the participants about the found bias for each specific travel time prediction.

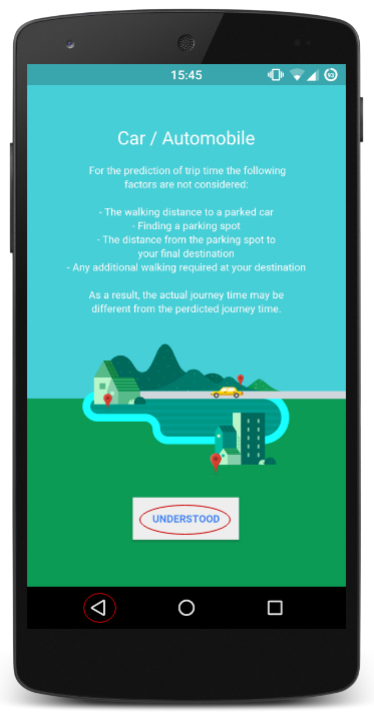

Figure 2. Warning label for driving by car

In order to implement our holistic concept of bias, we take different elements of the formula describing a holistic approach to bias into account:

- $\Delta$ User $=\bigcup(\alpha,(\beta \pm \gamma))$

where $\alpha$ represents User Intuition, $\beta$ represents Google Maps Recommendations and $\gamma$ represents User Error Correction.

We measured user intuition of travel time $(\alpha)$ by asking users for their estimates about distance and appropriate mode of transportation before showing them a mock-up of the Google Maps App. We measured Google Maps recommendations for travel time $(\beta)$ by using the Google Maps Android App at a specific time of day and writing down the recommendations. We measured user error correction of Google Maps recommendations $(\gamma)$ asking users how they make their decisions. Of these three concepts, the third is definitely the most difficult to measure, while the first and second can me more easily studied in detail.

\section{Analysis Results}

The extent to which biases are present in Google Maps (RQ3), needs to be understood in the context of the way data is presented in the Google Maps mobile app [21]. The most current version at this point in time was 9.79.0. Comparing the recommendations of Google Maps to real world data and the recommendations of others, we were able to identify numerous biases in the way Google Maps makes predictions. The identified biases can be conceptually grouped into four separate categories:

\subsection{Pseudo comparison}

The main challenge with biases in Google Maps stems from the way in which different types of transportation are compared and presented. As can be seen in Figure 3, Google Maps uses a "comparison bar" at the top of the screen, which suggests to the user that it is a fair comparison of different modes of transport.

This is because the public transportation trip time calculation and the walking trip time calculation, present a door to door calculation for the whole route from beginning to end (total trip time) while the driving and the taxi trip time calculation calculate trip time from the nearest road available to the users current location to the nearest road available at the users destination (partial trip time). Particularly in urban environments, the divergence between partial and total trip time for driving and taxi trip time calculation is likely to be considerable, as a large number of locations cannot be accessed by car. The comparison does not follow the relevant scientific literature from transportation sciences 
[31] and can be seen as an attempt to compare apples (total trip time for public transport and walking) to oranges (partial trip time for driving and taxis). Overall, the comparison bar presented by Google Maps to users suggests a comparison between variables, some of which are calculated as partial trip time and others which are calculated as total trip time and thus cannot be meaningfully compared. We thus call this comparison a pseudo comparison.

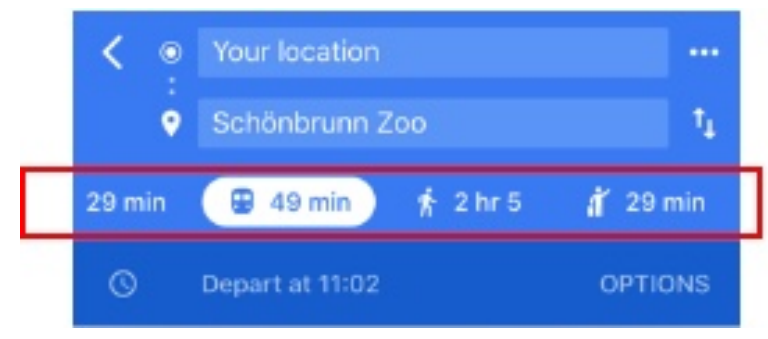

Figure 3. Google Maps comparison bar

\subsection{Data quality}

For a variety of reasons, the quality of the data on which the predictions of Google Maps in Vienna are based is low. To provide one example, a five year struggle over access to public transport data in a standardized Google format has resulted in low data quality of Google Maps for the city of Vienna [32]. However, Google has not communicated this issue to users. As Google is known for having access to large amounts of data, it is reasonable for users to conclude that they will receive very accurate estimates. The Google Maps App previously provided a range-based estimate to users (i.e., 12-26 minutes depending on traffic) [33]. In line with other HCI research on the value of "idea of representing uncertainty" [34], we believe that it is important to communicate this uncertainty to users. As a result, we have included these problems related to uncertainty into our warning labels.

\subsection{User planning}

Many estimates in Google Maps, assume some degree of user planning, but this is not communicated to to users. For instance, the estimated time for public transportation is not displayed immediately but rather at the most opportune moment for the next available connection. The necessary waiting time is not included in the calculation or shown in the comparison bar.

Similarly, the calculation for the taxi service (e.g., Uber, Lyft) does not include the necessary waiting time, which is therefore also not shown in the comparison bar. The user is only able to see the waiting time when specifically looking at the route for that particular option. Our analysis also revealed that opening the necessary App (e.g., Uber) to call the service, usually results in additional waiting time. In other words, Google Maps underestimates the necessary waiting time. It is also assumed that the user is standing next to a road and can be directly picked up by the taxi service.

Estimates for car driving time fail to include the time necessary for finding a parking spot, parking and walking from the parking spot to the final destination. It is assumed that the user considers and estimates this aspect on his own. In contrast, the internationally accepted way of measuring car travel time includes the time spent walking from the parking spot to the final destination [31].

\subsection{Universal accessibility}

When calculating an estimate for traveling by car, Google Maps assumes that the user has access to a private car. Furthermore, it is assumed that the user is already sitting in the car and can immediately start moving it. This assumption has significant implications, as the potential walking time to the car and the time required to move the car out of the garage or parking spot is not included in the estimation. This aspect of walking to the car is also included in the internationally accepted car travel time estimation [31].

Furthermore, Google Maps assumes that every destination is accessible or reachable by car. Even when its mapping algorithm shows that this is not the case, it fails to calculate the additional required walking time at the end of a car journey. However, this additional walking time is correctly added when using public transport or walking.

In summary, with regard to RQ1, the discovered biases lead to a significant and systematic underestimation of car journey time in Vienna. Furthermore, the necessary waiting time for taxi services or public transportation is not considered. Google does not communicate transparently that, in Vienna, travel time prediction is based on low-quality data. The comparison bar suggests to the user a fair comparison between different types of transportation modes, which is not the case.

While we believe that there is a compelling case that there is indeed a significant system bias $(\Delta$ System) in the recommendations presented by Google Maps $(\beta)$, we do not yet know what impact these recommendations have on users' bias ( $\Delta$ User). It is still possible that users have adapted to these biases such that they are able to correct for them and make accurate unbiased predictions. Thus, we cannot only ask whether bias is 


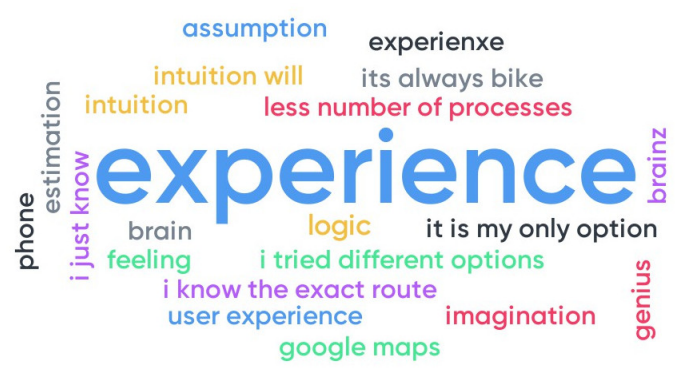

Figure 4. Basis for intuitive estimation

present in this information system; we also need to ask how this bias affects users.

\section{Pre-Study Results}

When study participants were asked to make an intuitive estimation, without using a digital tool, of the fastest mode of transportation to get from the classroom to the Vienna Zoo, most of them (17 out of 31) responded that taking public transportation is the fastest way to get there. Twelve participants were convinced that taking the car or taxi would be the fastest way. When asked how they estimated the fastest type of transportation, the most common answer was that they based their estimation on their "own experience." A follow-up discussion revealed that 30 out of 31 participants would normally use their phone to figure out the fastest mode of transportation.

Subsequently, participants were asked to make an estimation based on Google Maps. According to this, the majority of participants no longer believe that public transportation is the fastest option. Indeed, 16 out of 29 respondents were now convinced that taking the car or taxi is the fastest way to get to the Vienna Zoo. The minority (11 out of 29) still thought that public transport is the fastest option. Finally, when asked again how they choose the fastest type of transportation, only 4 out of 30 participants mentioned "experience" while 6 out of 30 explicitly mentioned a technical system (e.g., Google Maps, Apps).

In response to $\mathrm{RQ} 2$ regarding whether Google has an effect on how users perceive travel time, it seems clear that there is a substantial effect. This effect can be observed by looking at participants decision-making with regard to choosing transportation. There is also a strong suggestion of automation bias [29, 30], with participants trusting the systems and then blaming themselves for the errors made by the system.

\section{Online Survey Results}

Overall, 875 participants took part in the survey, but only 521 finished it and could be considered in our analysis. The participants mean age was 25.39 (SD $=7.67$ ), ranging from 17 to 65 years. On average, they have lived 9.88 (SD $=11.44$ ) years in Vienna and rated their general health, with $4.44(\mathrm{SD}=.63)$ on a 5-point likert scale. Of the 521 participants, $87.5 \%$ own a drivers license, $71.2 \%$ have a general public transportation ticket, $32.6 \%$ own a car and $68.3 \%$ possess a bike. Additionally, 22.6\% have a car-sharing membership (e.g., DriveNow) and $31.7 \%$ an account with a taxi company (e.g., Uber). When asked which App participants use for traveling in Vienna, 47.7\% ranked Google Maps the highest, followed by $25.4 \%$ for Qando and 19.4\% for OEBB Scotty; the remaining 7.5\% picked other Apps. On a 5-point likert scale, participants rated their mean experience in using Google Maps with 4.32 (SD = 1.20), their confidence in the accuracy of mapping data with $4.00(\mathrm{SD}=1.09)$, their perceived quality of suggested routes with 3.83 (SD = 1.04). Participants had a general trust in Google Maps of 3.85 ( $\mathrm{SD}=1.04)$. Overall, on a 5-point likert scale, participants had a mean confidence that they would reach the destination without Google Maps of 3.46 (SD $=1.04$ ) for all routes combined.

To illustrate the shift in decision-making produced by Google Maps, we have provided in Figure 5 an overview of the shifts in transportation choice before and after using Google Maps for the first route. This shows a strong shift from intuitive decisions - where users overwhelmingly favor public transport before using Google Maps — and decisions made after using Google Maps, where the 'best' form of transportation is far more likely to be 'driving' than was previously the case. 


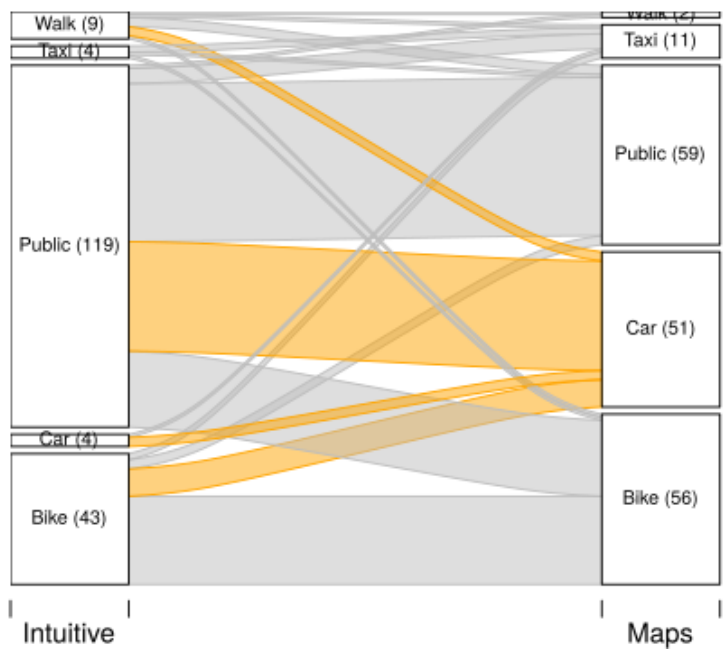

Figure 5. Overview of changes in user decision making for route 1 before and after using Google Maps

As our analysis and pre-study hints toward a bias in Google Maps to use an automobile (car or taxi), we assessed the change in proportion of participants to use either an automobile or an other mode of transportation (public transport, walk or bike) using the McNemar's test. This test revealed a statistically significant difference in the proportion of participants $(n=183$ in this condition) choosing an automobile intuitively and based on Google Maps for the first $\left(\mathrm{p}_{i}\right.$.001) and second ( $p ; .001)$ route. For the first route, only $4.9 \%$ of the participants intuitively chose an automobile, but after seeing the Google Maps mockup 34.6\% of them chose an automobile as the best type of transportation. For the second route there is a similar result, with $6.6 \%$ of the participants intuitively choosing an automobile and $39.1 \%$ choosing an automobile after consulting Google Maps.

To analyze the effects the proposed accountability mechanisms have on the chosen type of transportation, a cross-table with Pearson's chi square was used. A significant association between the accountability mechanism and the type of transportation participants chose was found for the first route $\chi^{2}(8)=41.06, p ; .001$ and the second route $\chi^{2}(8)=66.14, p ; .001$. For the first route a follow-up z-test with Bonferroni adjustment revealed that for the "Warning Label Version" more participants than expected chose the car $(\mathrm{z}=2.6)$. In contrast, for the "Unbiased Version" fewer participants than expected chose the car $(\mathrm{z}=-4.1)$ while more than expected chose the bike $(\mathrm{z}=2.2)$. For the second route, significantly more participants than expected chose the car in the "Original Version" $(\mathrm{z}=2.5)$ as well as the
"Warning Label Version" ( $\mathrm{z}=2.5)$. For this route, in the "Unbiased Version", significantly fewer participants chose the car $(\mathrm{z}=-4.9)$ or a taxi service $(\mathrm{z}=-2.1)$ and more than expected chose public transportation $(z=3.1)$. The exact results for the second route can be seen in Table 1. Based on the odds ratio for the second route, the odds of a participant choosing a car were 7.6 times higher if he uses the "Original Version" of Google Maps compared to the "Unbiased Version". A final analysis of the effect of the accountability mechanism on trust in Google Maps revealed no significant effect.

\begin{tabular}{|l|c|c|c|c|c|}
\hline \multirow{2}{*}{ Version } & \multicolumn{5}{|c|}{ Type of Transportation } \\
\cline { 2 - 6 } & Car & Public & Walk & Taxi & Bike \\
\hline Count & 60 & 58 & 4 & 10 & 47 \\
$\%$ in Type & $48.8 \%$ & $27.4 \%$ & $50.0 \%$ & $45.5 \%$ & $33.1 \%$ \\
Std. Residual & $2.5 *$ & -1.9 & .7 & .8 & -.4 \\
\hline Warning & 52 & 54 & 1 & 11 & 34 \\
Count & $42.3 \%$ & $25.5 \%$ & $12.5 \%$ & $50.0 \%$ & $23.9 \%$ \\
$\%$ in Type & $2.5 *$ & -1.2 & -.9 & 1.7 & -1.4 \\
Std. Residual & & 100 & 3 & 1 & 61 \\
\hline Unbiased & 11 & 100 & & \\
Count & & & & & \\
\% in Type & $8.9 \%$ & $47.2 \%$ & $37.5 \%$ & $4.5 \%$ & $43.0 \%$ \\
Std. Residual & $-4.9 * * *$ & $3.1 * *$ & .1 & $-2.4 *$ & 1.7 \\
\hline
\end{tabular}

Table 1. Cross-tabulation for the second route (* z-score is significant at the .05 level; ${ }^{* *}$ at the .01 level; ${ }^{* * *}$ at the .001 level)

\section{Discussion}

RQ 1: Are there any algorithmic bias in Google Map when used in Vienna? Based on our analysis it seems clear that that there is indeed a $\Delta$ System bias within Google Maps. The effect of this bias on Google Maps predictions is systematic underestimation of the time needed for driving due to the exclusion of relevant variables from the prediction algorithm that calculates travel distance while excluding these variables for public transport. For example, by only calculating driving distance on a route which requires additional walking at the beginning and end of the trip to get to a final destination, Google Maps often underestimates car travel time in Vienna (partial trip time). By contrast, additional walking time is included by Google Maps as part of public transport journey time for estimates on the same route (total trip time). This and other related biases suggest to users that driving is faster than it actually is, effectively making driving or taking a taxi seem like faster and therefore more attractive transportation options than they actually are.

While this bias exists all around the world, it only 
manifests itself in large urban areas with good public transport, where the bias is likely to exhibit a strong effect. This is because urban areas have far more pedestrian areas than comparable rural areas and finding parking is comparably difficult, while public transport provides a viable alternative. As such, the bias is most likely to have an effect in urban multi-modal transportation settings in which users actually have several viable alternatives and choose the best option from them [35]. Importantly, urban multi-modal transportation settings are also one of the most common areas where Google Maps is used, as it is typically one of the few apps that provides some degree of comparison between different forms of transportation in an urban setting.

Based on our analysis it seems reasonable to suggest that there are distinct $\Delta$ System biases in Google Maps. This finding is consistent with other research on mapping technologies which suggests that mapping technologies inherently cannot be perfect and are likely to contain some form of bias in specific usage contexts $[5,1,2]$.

RQ 2: How strong is the effect of Google Maps on how users perceive travel time in Vienna? That $\Delta$ System bias has an effect on users' perception of travel time is clearly displayed in the pre-study. Participants in the pre-study and online survey were better at making accurate predictions of distance intuitively without using Google Maps. Despite intuitively knowing that public transport was likely to be faster on the route provided to them, users developed answers to the questions that followed the information provided to them by the Google Maps App and assumed that driving would be the faster option. Rather than following the information that they intuitively knew to be correct, users preferred to trust Google.

This trust in Google Maps is also present in the online survey. Our participants display high confidence in the materials used to generate Google Maps, the quality of the suggested routes and a general trust in Google Maps. Even after being shown one of our warning labels about bias in Google Maps, users trust in Google Maps and its recommendations remains high, with no statistical effect of being shown a warning label. Thus, it seems clear to us that $\Delta$ System bias does indeed have an effect on $\Delta$ User bias which directly influences user perceptions of travel time. This finding, which is present in both the pre-study and the online survey, is consistent with literature on automation bias [29, 30] and more broadly literature on the powerful effects on human behavior of socio-technical systems [36, 37, 38].

RQ 3: How strong is the effect of Google Maps on users' choice of best type of transportation in
Vienna? Google Maps has a systematic bias towards driving or taking a Taxi, Uber or Lyft. For the first and second route in our online survey only $4.9 \%$ and $6.6 \%$ of the participants wanted to use an automobile, but after seeing the Google Maps mockups 34.6\% and 39.1\% of participants respectively want to use an automobile. For the third route, there is no significant effect. We believe this result could be due to specific routing conditions for this route in Vienna.

Based on the results of the pre-study and the online survey, we believe that there is a strong argument that bias in Google Maps influences users choice of the best type of transportation. As a result, we believe that there is a significant effect of $\Delta$ System bias on $\Delta$ User bias. While further research will be required to disaggregate which types of errors in Google Maps users are able to correct for $(\gamma)$, it seems clear that their error correction is not sufficient to prevent the bias from influencing their choice of best type of transportation. This result is consistent with existing HCI research on users difficulty in factoring in uncertainty into travel decision-making without design elements that support the visualization of uncertainty [34], as well as research on cognitive load theory [39].

Moreover, our analysis suggests that presentation of mapping results matters a great detail. When presented with greater transparency - even when they are constructed as warning labels - are not effective at informing users about bias in Google Maps. This could be because users are used to clicking through all manner of warnings, terms of service and cookie banners already, and thus even yellow warning labels draw little attention or interest. By contrast the process of providing an unbiased version of Google Maps based on ground truth data has a significant effect. Users of the unbiased version of Google Maps are much less likely to drive and much more likely to take public transport.

Notably the effect is strongest for decisions to take an automobile, as this is the mode of transportation where the strongest bias is present. Here Google Maps clearly seems to be nudging users towards driving. Once the effects of this nudging are removed, users are less likely to travel by car than was previously the case. This systematic bias is particular evident for second route in the online survey, with the odds of the participants choosing a car as the best option 7.6 times higher when using Google Maps predictions rather than ground truth data.

Thus we believe that there is a strong indication that system error correction provides a far better mechanism to correct bias than transparency which takes place through warning labels. This is consistent with literature on transparency which questions the 
efficacy of transparency as an accountability mechanism $[40,41,42]$.

\section{Conclusion and Limitations}

This paper has shown that for the routes we tested in Vienna, there is a significant bias in the estimations made by Google Maps in a specific limited set of routes. These bias influences users' perception of travel time and their choice of transportation medium. As the study population consisted of young students who are on average in their mid-20s and is not representative, we cannot generalize these findings to everyone in Vienna or to other similarly large European cities.

It should also be noted that due to the habitual nature of choices of transportation, the effects of our research on decision-making are likely to be particularly strong on multi-modal transport users, who are able to choose between multiple different forms of transport [35, 43]. Further research is required to fully understand the effect this has on mobility decision-making, in particular with regard to the modal split of mobility users [?] and the associated environmental impact of nudging users towards driving.

Despite these limitations, we believe that the paper presents an interesting and useful case study on the limitation of geographic information systems in urban environments like Vienna. We have also shown that these limitations have effects on user behavior, in particular when it comes to choosing driving over other forms of transport. As much of this bias are systematic inappropriate comparisons of trip time estimates in Google Maps, it is possible that these effects extend beyond the three routes which we tested. Whether this effect holds in other settings or not will require further research to understand this phenomenon in greater detail, as well as unpacking the cognitive models of Google Maps users.

\section{References}

[1] J. B. Harley, "Maps, knowledge, and power," Geographic thought: a praxis perspective, pp. 129-148, 2009.

[2] H. Lin and M. Batty, "Virtual geographic environments: a primer," Virtual Geographic Environments. Science Press, Beijing, pp. 1-10, 2009.

[3] D. R. Montello, "Behavioral and cognitive geography: introduction and overview," in Handbook of Behavioral and Cognitive Geography, pp. 3-15, Edward Elgar Publishing, 2018. Google-Books-ID: 8ihWDwAAQBAJ.

[4] M. J. Egenhofer, K. C. Clarke, S. Gao, T. Quesnot, W. R. Franklin, M. Yuan, and D. Coleman, "Contributions of GIScience over the past twenty years," Advancing Geographic Information Science: The Past and the Next Twenty Years, pp. 9-34, 2016.
[5] G. Quattrone, L. Capra, and P. De Meo, “There's no such thing as the perfect map: Quantifying bias in spatial crowd-sourcing datasets," in Proceedings of the 18th ACM Conference on Computer Supported Cooperative Work \& Social Computing, pp. 1021-1032, ACM, 2015.

[6] B. Hecht and D. A. Shamma, "A Crash Course in Modern Geography for HCI Researchers and Practitioners," in Proceedings of the Extended Abstracts of the 32Nd Annual ACM Conference on Human Factors in Computing Systems, CHI EA '14, (New York, NY, USA), pp. 1031-1032, ACM, 2014. event-place: Toronto, Ontario, Canada.

[7] J. Schoening, B. Hecht, and W. Kuhn, "Informing Online and Mobile Map Design with the Collective Wisdom of Cartographers," in Proceedings of the 2014 Conference on Designing Interactive Systems, DIS '14, (New York, NY, USA), pp. 765-774, ACM, 2014. event-place: Vancouver, BC, Canada.

[8] C. M. Dalton, "Sovereigns, Spooks, and Hackers: An Early History of Google Geo Services and Map Mashups," Cartographica: The International Journal for Geographic Information and Geovisualization, vol. 48, pp. 261-274, Dec. 2013.

[9] B. Friedman and H. Nissenbaum, "Bias in Computer Systems," ACM Trans. Inf. Syst., vol. 14, pp. 330-347, July 1996.

[10] D. R. Montello, "The perception and cognition of environmental distance: Direct sources of information," in Spatial Information Theory A Theoretical Basis for GIS (S. C. Hirtle and A. U. Frank, eds.), vol. 1329, pp. 297-311, Berlin, Heidelberg: Springer Berlin Heidelberg, 1997.

[11] R. Colledge, "Cognitive approaches to the analysis of spatial behavior," Environment and cognition, pp. 59-94, 1973.

[12] A. Stevens and P. Coupe, "Distortions in judged spatial relations," Cognitive psychology, vol. 10, no. 4 , pp. 422-437, 1978.

[13] R. Alt, S. Human, and G. Neumann, "End-user empowerment in the digital age," in Proceedings of the 53rd Hawaii International Conference on System Sciences, pp. 4099-4101, 2020.

[14] S. Human, R. Gsenger, and G. Neumann, "End-user empowerment: An interdisciplinary perspective," in Hawaii International Conference on System Sciences 2020, pp. 4102-4111, 2020.

[15] D. M. Mark, C. Freksa, S. C. Hirtle, R. Lloyd, and B. Tversky, "Cognitive models of geographical space," International journal of geographical information science, vol. 13, no. 8, pp. 747-774, 1999.

[16] B. Guo and S. Zhou, "Re-examining the role of attitude in information system acceptance: a model from the satisfaction-dissatisfaction perspective," Enterprise Information Systems, vol. 10, no. 4, pp. 444-466, 2016.

[17] S. Peer, J. Knockaert, P. Koster, and E. T. Verhoef, "Over-reporting vs. overreacting: Commuters perceptions of travel times," Transportation Research Part A: Policy and Practice, vol. 69, pp. 476-494, 2014.

[18] S. Peer, J. Knockaert, and E. T. Verhoef, "Train commuters' scheduling preferences: Evidence from a large-scale peak avoidance experiment," Transportation Research Part B: Methodological, vol. 83, pp. 314-333, 2016. 
[19] B. Hecht and M. Stephens, "A tale of cities: Urban biases in volunteered geographic information," in Eighth International AAAI Conference on Weblogs and Social Media, 2014.

[20] B. Hecht and D. Gergle, "Measuring Self-focus Bias in Community-maintained Knowledge Repositories," in Proceedings of the Fourth International Conference on Communities and Technologies, C\&T '09, (New York, NY, USA), pp. 11-20, ACM, 2009. event-place: University Park, PA, USA.

[21] B. Wagner, T. Winkler, and S. Human, "Comparing Travel Time Estimates of Routing Applications in Vienna," in Workshop on Engineering Accountable Information Systems, European Conference on Information Systems (ECIS 2019), (Stockholm, Sweden), 2019.

[22] M. Veale, M. Van Kleek, and R. Binns, "Fairness and Accountability Design Needs for Algorithmic Support in High-Stakes Public Sector Decision-Making,' arXiv:1802.01029 [cs], pp. 1-14, 2018. arXiv: 1802.01029

[23] Z. Tufekci, J. C. York, B. Wagner, and F. Kaltheuner, "The Ethics of Algorithms: from radical content to self-driving cars," tech. rep., European University Viadrina, Berlin, Germany, 2015.

[24] Z. Tufekci, "Algorithms in our Midst: Information, Power and Choice when Software is Everywhere," in Proceedings of the 18th ACM Conference on Computer Supported Cooperative Work \& Social Computing, (Vancouver, BC, Canada), pp. 1918-1918, Proceedings of the 18th ACM Conference on Computer Supported Cooperative Work \& Social Computing, 2015.

[25] S. Human and F. Cech, "A human-centric perspective on digital consenting: The case of gafam," in Human Centred Intelligent Systems (A. Zimmermann, R. J. Howlett, and L. C. Jain, eds.), (Singapore), pp. 139-159, Springer Singapore, 2021.

[26] S. Human, G. Neumann, and M. F. Peschl, "[how] can pluralist approaches to computational cognitive modeling of human needs and values save our democracies?," Intellectica, no. 70, pp. 165-180, 2019.

[27] S. Human, G. Bidabadi, and V. Savenkov, "Supporting pluralism by artificial intelligence: Conceptualizing epistemic disagreements as digital artifacts," in Philosophy and Theory of Artificial Intelligence 2017 (V. C. Müller, ed.), (Cham), pp. 190-193, Springer International Publishing, 2018.

[28] S. Human, F. Fahrenbach, F. Kragulj, and V. Savenkov, "Ontology for representing human needs," in Knowledge Engineering and Semantic Web (P. Różewski and C. Lange, eds.), (Cham), pp. 195-210, Springer International Publishing, 2017.

[29] M. T. Dzindolet, S. A. Peterson, R. A. Pomranky, L. G. Pierce, and H. P. Beck, "The role of trust in automation reliance," International journal of human-computer studies, vol. 58, no. 6, pp. 697-718, 2003.

[30] L. J. Skitka, K. L. Mosier, and M. Burdick, "Does automation bias decision-making?," International Journal of Human-Computer Studies, vol. 51, no. 5, pp. 991-1006, 1999.

[31] I. Benenson, K. Martens, Y. Rofe, and A. Kwartler, "Public transport versus private car GIS-based estimation of accessibility applied to the Tel Aviv metropolitan area," The Annals of Regional Science, vol. 47, no. 3, pp. 499-515, 2011.
[32] B. Riegler, "Warum Google Maps die Wiener so viel zu Fuss gehen laesst," 2017.

[33] B. Schwartz, "Google Maps Removes Driving With Traffic Estimates," 2011.

[34] M. Kay, T. Kola, J. R. Hullman, and S. A. Munson, "When (ish) is My Bus?: User-centered Visualizations of Uncertainty in Everyday, Mobile Predictive Systems," in Proceedings of the 2016 CHI Conference on Human Factors in Computing Systems - CHI'16, (Santa Clara, California, USA), pp. 5092-5103, ACM Press, 2016.

[35] A. U. Raghunathan, D. Bergman, J. Hooker, T. Serra, and S. Kobori, "Seamless Multimodal Transportation Scheduling," arXiv:1807.09676 [math], July 2018. arXiv: 1807.09676.

[36] C. O'Neil, Weapons of Math Destruction: How Big Data Increases Inequality and Threatens Democracy. New York: Crown, Sept. 2016.

[37] F. Pasquale, The Black Box Society: The Secret Algorithms That Control Money and Information. Cambridge, Massachusetts: Harvard University Press, 2015.

[38] R. M. Bond, C. J. Fariss, J. J. Jones, A. D. I. Kramer, C. Marlow, J. E. Settle, and J. H. Fowler, "A 61-million-person experiment in social influence and political mobilization," Nature, vol. 489, no. 7415 , pp. 295-298, 2012.

[39] N. Hollender, C. Hofmann, M. Deneke, and B. Schmitz, "Integrating cognitive load theory and concepts of human-computer interaction," Computers in Human Behavior, vol. 26, no. 6, pp. 1278-1288, 2010.

[40] C. Payne, "On the security of open source software," Information systems journal, vol. 12 , no. 1, pp. 61-78, 2002.

[41] J.-H. Hoepman and B. Jacobs, "Increased security through open source," Communications of the ACM, vol. 50, no. 1, pp. 79-83, 2007.

[42] H. Plate, S. E. Ponta, and A. Sabetta, "Impact assessment for vulnerabilities in open-source software libraries," arXiv:1504.04971 [cs], Apr. 2015. arXiv: 1504.04971.

[43] F. Huelsmann, W. Zimmer, G. Sunderer, and K. Goetz, "share: Wissenschaftliche Begleitforschung zu car2go mit batterieelektrischen und konventionellen Fahrzeugen," tech. rep., Institute for Applied Ecology, Freiburg, Germany, 2018. 